Brit. J. vener. Dis. (1957), 33, 5.

\title{
COMPARATIVE STUDIES ON THE HISTO-PATHOLOGY OF SYPHILIS, YAWS, AND PINTA*†
}

\author{
BY \\ C. M. HASSELMANN+ \\ From the Universitäts-Hautklinik, Erlangen
}

The mass campaigns against treponemal diseases in various countries undertaken by the United Nations World Health Organization (WHO) and the United Nations International Children's Emergency Fund (UNICEF) together with the respective national governments have again focused interest on the epidemiology, mode of transmission and infection, clinical manifestations, and control and treatment, of these treponematoses, viz. syphilis, framboesia tropica (yaws), and pinta. The magnitude of the various yaws control programmes in the world is illustrated by the fact that more than 60 million people have been examined and over 10 million treated. In Indonesia alone, where the largest programme is under way, the figures show that, during the first survey, between June 1950, and June 1956, over 20 million people were examined out of an estimated total population of 27 million in the campaign areas, and a little more than 2.9 million cases of yaws were treated. During the second survey nearly 23 million people were examined, and a little more than one million treated. Although in South-East Asia and the Pacific Islands the incidence of yaws varies greatly from one country to another, the average seems still to be about 10 per cent. for the first survey, with my own data collected in Indonesia in 1950 to 1951 indicating an overall incidence of 17.7 per cent. in the different target areas then under survey. When assessing the yaws campaign in the Philippines in 1952, I found an incidence of less than 10 per cent. in the target areas, with cumulative data indicating nearly 200,000 cases treated out of about 3.5 million examined (i.e. about $5 \cdot 7$ per cent.).

Apart from their respective causative treponemes (Treponema pallidum of syphilis, Treponema pertenue of yaws, and Treponema pinta Herrejoni of pinta)

\footnotetext{
* Received for publication December 1956

+ Paper given at the 7 th International Congress of Comparative Pathology, Lausanne, Switzerland, 1955.

$\mp$ Formerly W.H.O. Chief Medical Adviser to the Treponematosis Control Programme, Indonesia, and Special Consultant to the Philippine Treponematosis Control Programme.
}

and different modes of transmission, the various clinical lesions typical of each of these three treponemal diseases are conditioned by their distinctive behaviour in the host's tissues.

This precise "histio-biotropism" governs, in its turn, both the morphology and the histo-pathology of the various clinical manifestations, as established by human and animal experimental evidence (Goodpasture, 1923; Sellards, Lacy, and Schöbl, 1926; Schöbl, Sellards, and Lacy, 1926; Schöbl and Sellards, 1926; Schöbl, 1928; Schöbl and Hasselmann, 1932; Hasselmann, 1930, 1931, 1932, 1933, 1938a,b, 1949, 1951, 1952, 1953a,b,c, 1955; Turner, 1937; Turner, Hollander, and Schaeffer, 1953; McLeod and Turner, 1946; Hollander and Turner, 1955).

\section{Syphilis}

Whereas the primary indurated papular lesion in syphilis may soon progress into the well-known sharply punched-out ulcer, or "hard chancre", the initial framboetic papular lesion soon grows into a papilloma, without any tendency to necrosis at this early stage of the yaws infection.

In syphilis, the usual necrosis of the primary indurated papular lesion leading to the typical hard chancre is obviously due to the direct toxic action of $T$. pallidum. The epidermis proper may show only moderate acanthosis and spongiosis. In the cutis, rather sharply outlined infiltrations consist of round cells, fibroblasts, and histiocytes, some of which may be already of the epithelioid type, plasma cells, some eosinophils, and even occasional giant cells. In contrast to the plasma cells often dominating the cellular infiltrates in yaws, the plasma cells in syphilis occasionally show marked evidence of hyaline degeneration. The most conspicuous features in syphilis, both in the primary papular stage and chancre as well as in the secondary syphilides, are the frequent pronounced inflammatory changes in the intima and media of the capillaries, with cellular 
infiltration, endothelial proliferation, and even obliteration of the lumina (Figs 1-4).

(1)
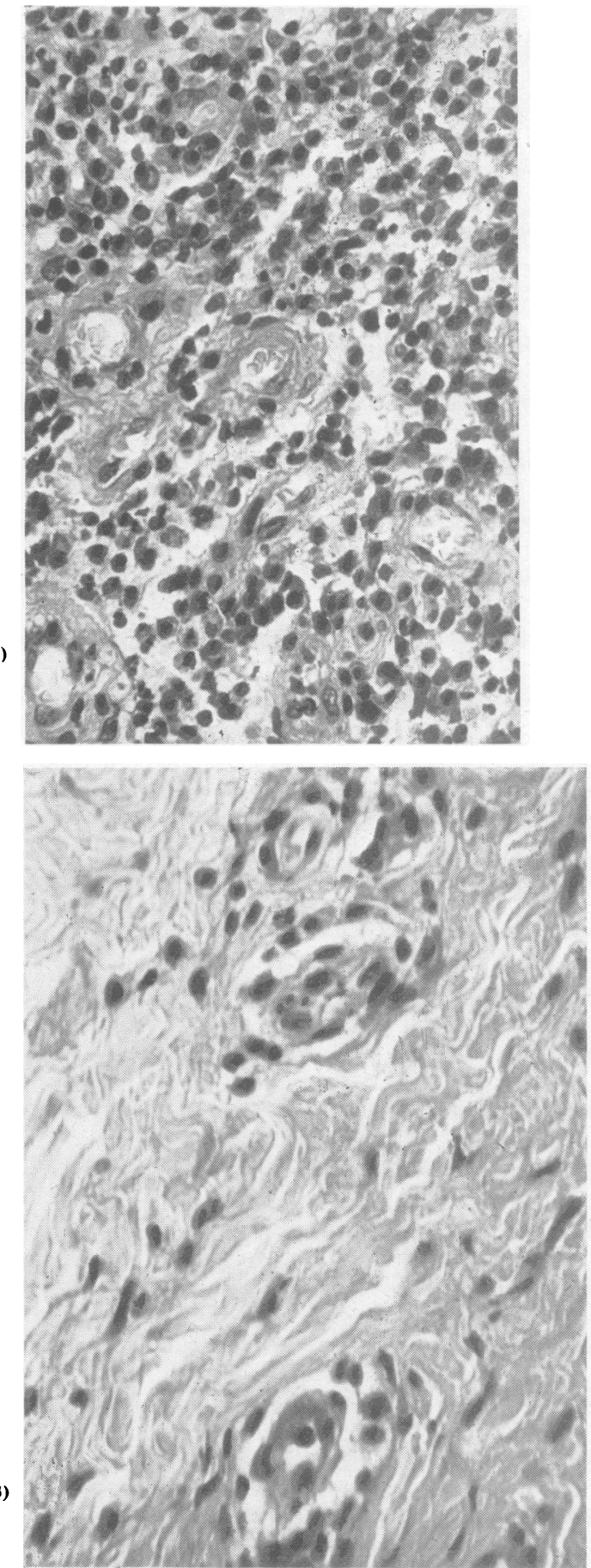

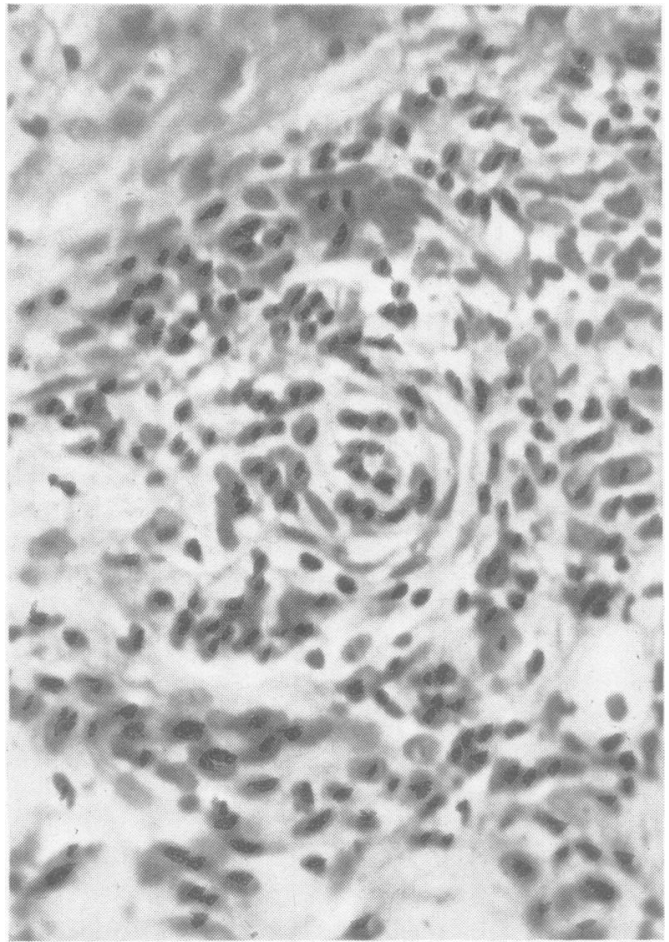

(2)

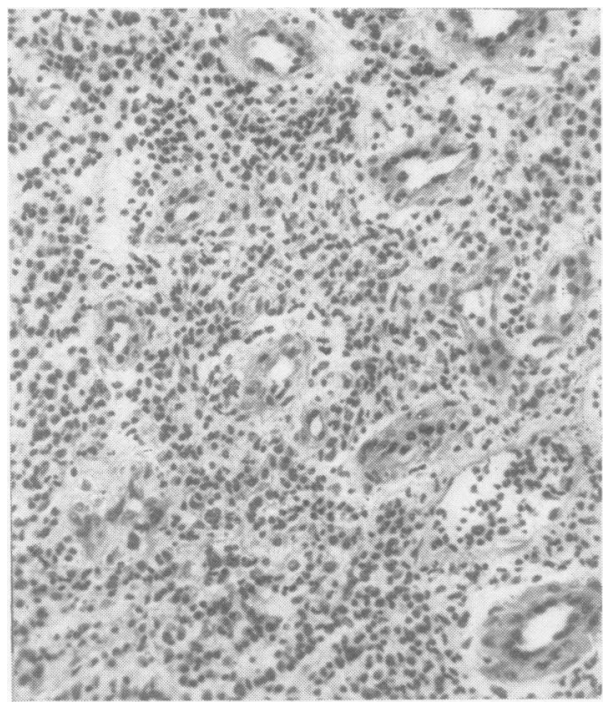

Fig. 1.-Perigenital syphilitic papule (Condyloma latum). Proliferation of intima and media with partial obliteration, cellular infiltrate. (All sections stained by haematoxylin and eosin.)

Fig. 2.-Syphilitic papule with extensive perivascular infiltrates, and vascular pathology with partial obliteration of lumen.

Fig. 3.-Syphilitic papule: extensive vascular pathology with partial obliteration of the lumen.

Fig. 4.-Late tubero-serpiginous syphilide. Massive vascular pathology. 


\section{Yaws}

In contrast, the initial framboetic papule soon grows into a papilloma (Figs 5, 6, and 7), nearly always with an exuberant inflammatory response in the epidermis and cutis. The characteristic histo-pathological architecture both of the initial yaws papule, or papilloma, and of the metastatic generalized polypapillomata is the usually enormous acanthosis of the epidermis proper, and the severe inflammatory changes in corium and cutis with the common appearance of a "plasmocytoma". Hyperkeratosis and parakeratosis soon cannot be seen because of the early loss of the stratum corneum and the stratum granulosum, respectively. The stratum spinosum shows very marked broadening of its entire width, usually an enormous downgrowth of the rete pegs into the cutis and even into the subcutis. Intra- and inter-cellular oedema is marked, with occasional vacuolization and degeneration, including disappearance of pigment. Often, diapedesis of neutrophils may lead to the formation of miliary intra-epidermal micro-abscesses, so typical of yaws. However, so-called "epidermiolysis" may be present, but without the otherwise typical "dropped-off" or hazy, "moth-eaten" phenomenon encountered, e.g. in lichen ruber planus. The inflammatory response of the tissues may be so marked that clinically the epidermal surface is lost, serum oozes out, and a non-adherent, yellowish crust results, consisting of dried serum and epidermal scales covering the soft, non-indurated papilloma.

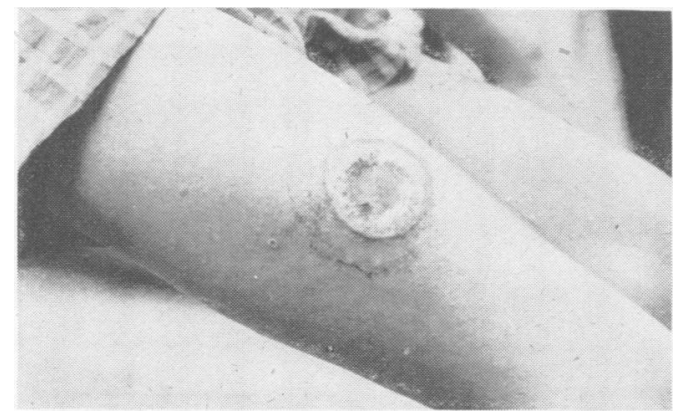

Fig. 5.-Initial so-called "primary" mother-yaws papilloma with

This usually enormous inflammatory pathology in the corium and cutis attests to the acute response of these mesodermal parts. Both stratum papillare and stratum reticulare show an extremely loose cellular infiltration, markedly oedematous, with predominating plasma cells, fibroblasts, and histiocytes, some of which are already of the epithelioid type, with round cells, and a few eosinophils; giant

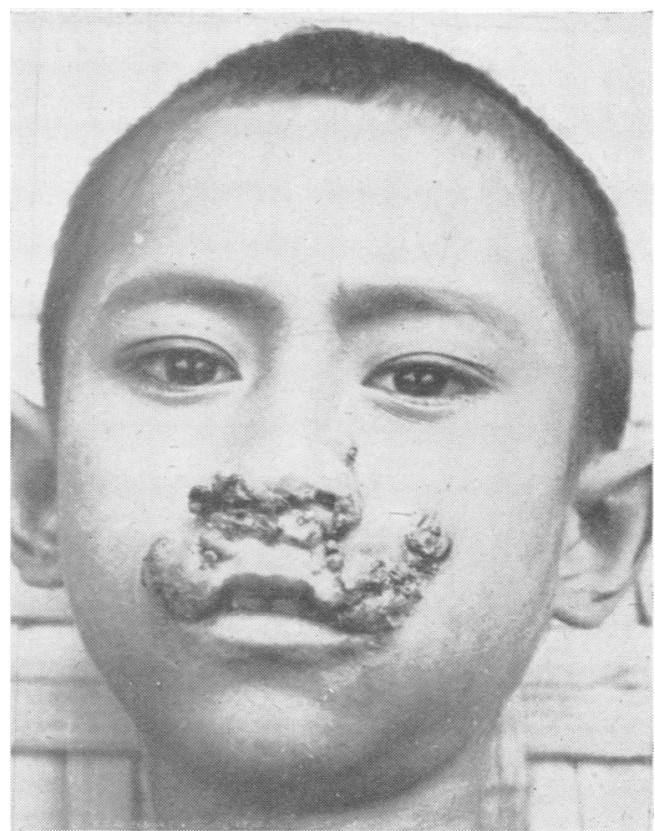

Fig. 6.-Polypapillomata framboesica: these lesions around the facial orifices might easily lead to rhinopharyngitis mutilans ("gangosa") through obvious allergic exacerbation.

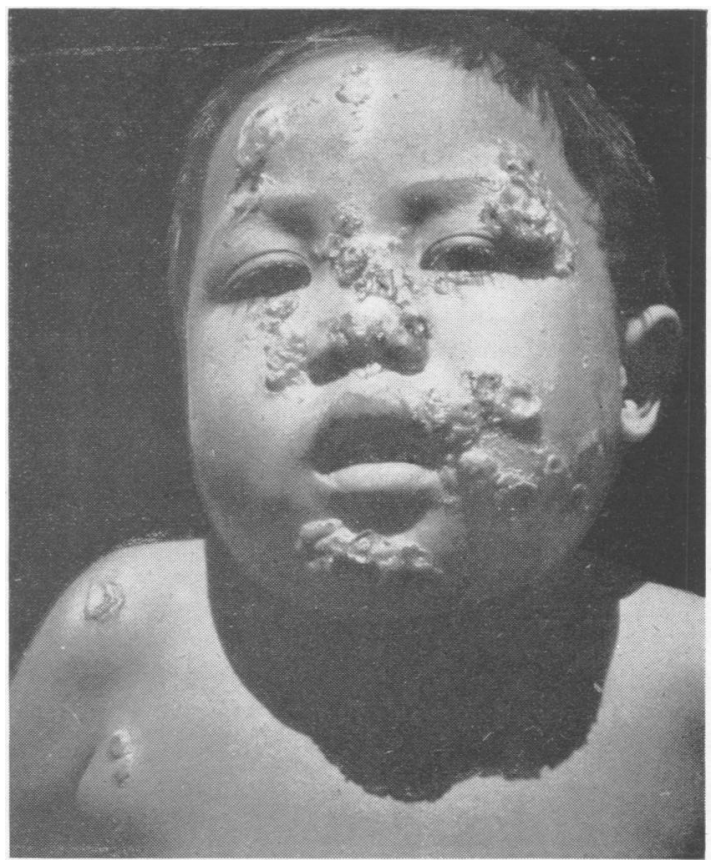

Fig. 7.-Polypapillomata of the metastatic generalized stage of yaws. 
BRITISH JOURNAL OF VENEREAL DISEASES

cells are rarely encountered. In this typical "plasmocytoma" the blood vessels are not affected, either in the initial yaws papule or papilloma, or in the metastatic polypapillomatous efflorescences. Intima and media remain quite intact, there being neither cellular infiltration nor endothelial proliferation (Figs 8, 9, 10, 11, 12, 13). No coagulative necrosis

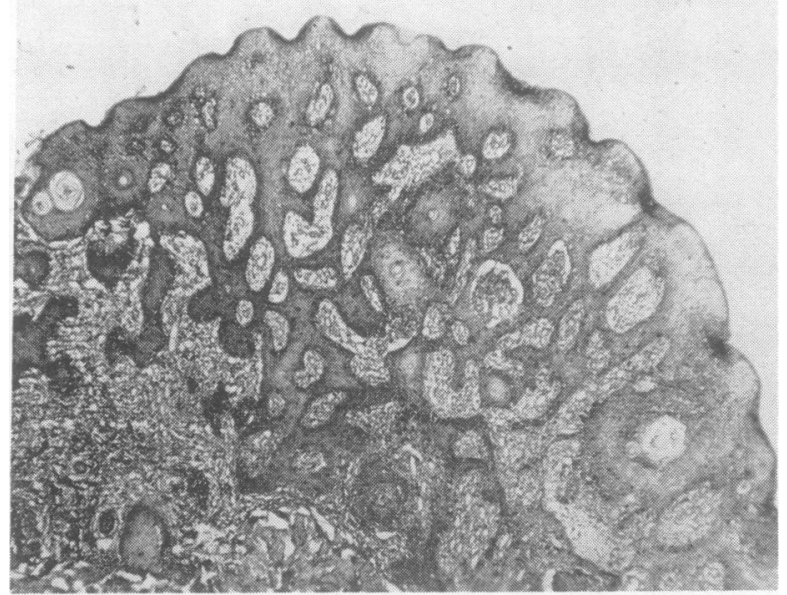

Fig. 8.-Yaws papilloma. Enormous acanthosis and loose cellular infiltrate in corium and cutis, mainly of plasma cells ("Plasmocytoma").

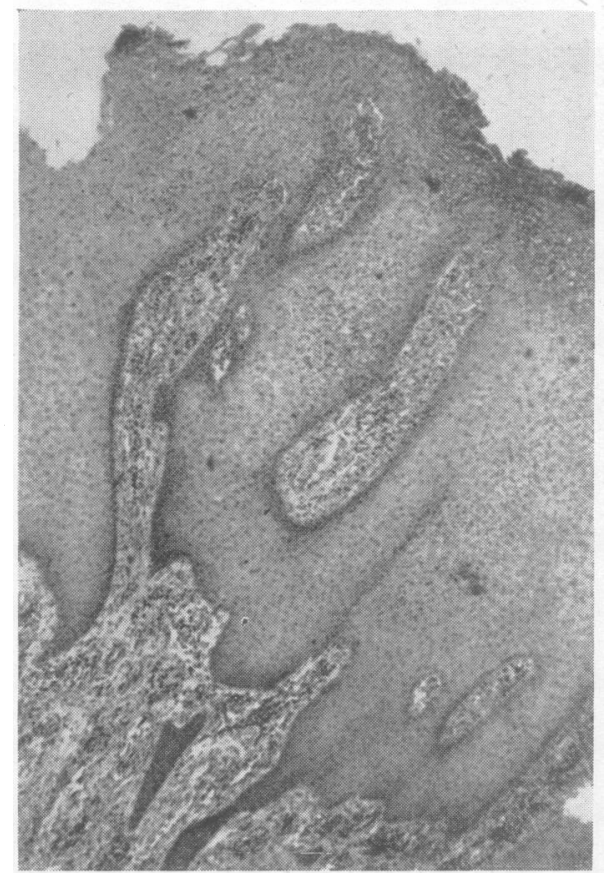

Fig. 10.-Yaws papilloma: medium magnification. Migration of neutrophils through epidermis; formation of intra-epidermal microabscesses. No vascular pathology of intima or media.

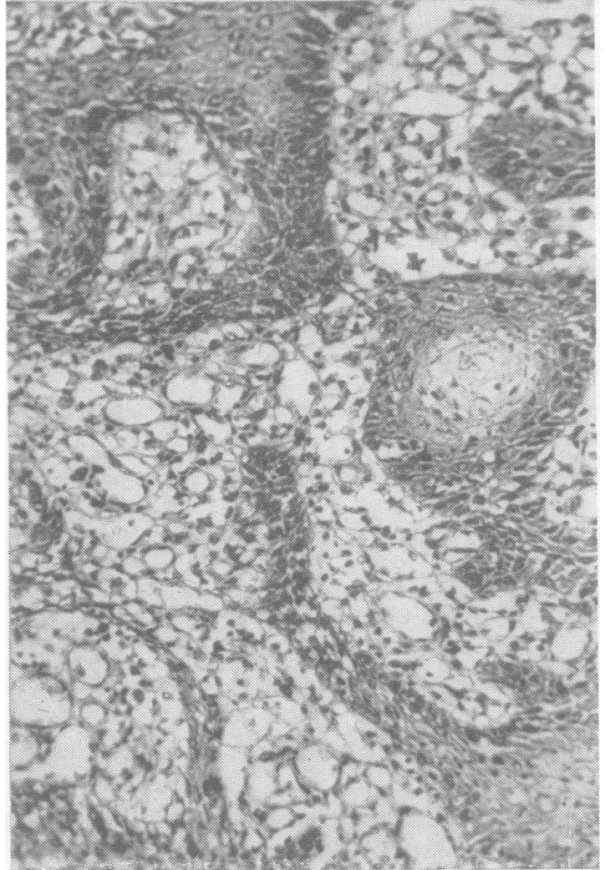

Fig. 9.-High magnification of Fig. 8. Extremely loose cellular infiltrate of corium and cutis with many plasma cells. No pathology of intima or media.

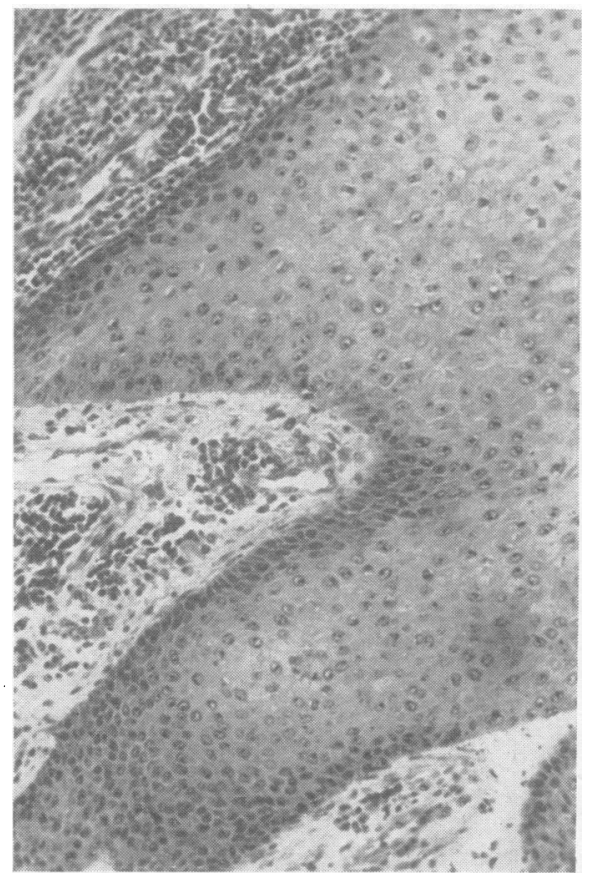

Fig. 11.- - High magnification of Fig. 10 showing perivascular infiltrations, but with normal intima and 
occurs and consequently no ulcer formation results. sensitization has developed to such an extent that Experimental evidence appears to suggest that only any sudden increase of antigen may enhance an in the very late stage of framboesia tropica does sudden necrosis with ulceration set in, if and when

apparent allergic exacerbation of pre-existing skin lesions.

Fig. 12.-High magnification of Fig. 11. Perivascular infiltrate with plasma cells, fibroblasts and histiocytes, some being of the epithelioid type. No vascular pathology.
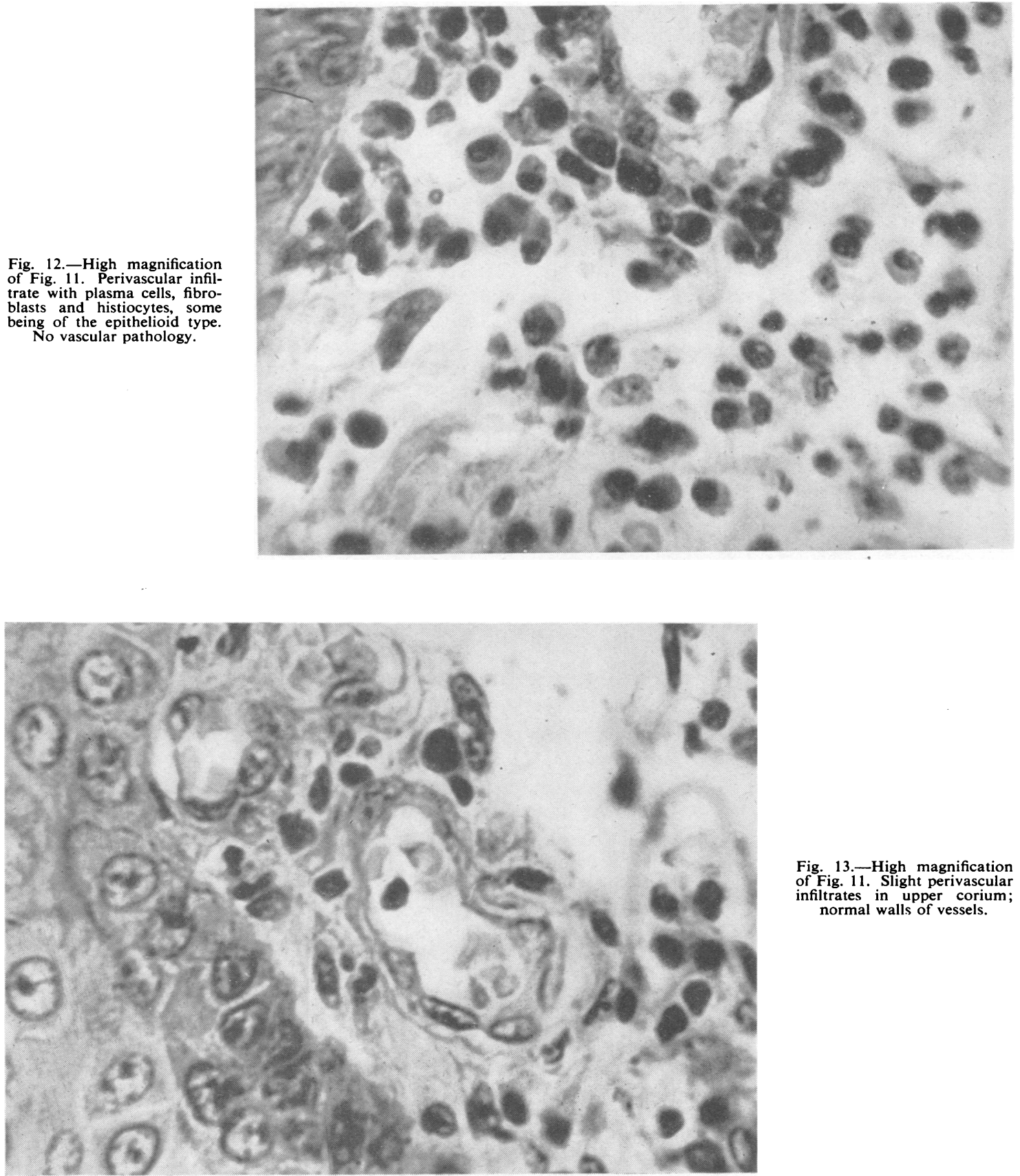

Fig. 13-High magnification of Fig. 11. Slight perivascular infiltrates in upper corium; normal walls of vessels. 
In the field and dispensaries, patients with ulcerations may be numerous, and it always remains difficult to ascertain the exact pathogenesis in these late yaws cases. Experimentally, Schöbl and others (1928) succeeded in reproducing in the Philippine Macaccus cynomolgus all clinical stages of yaws including ulcerative rhinopharyngitis mutilans ("gangosa") (Fig. 14).

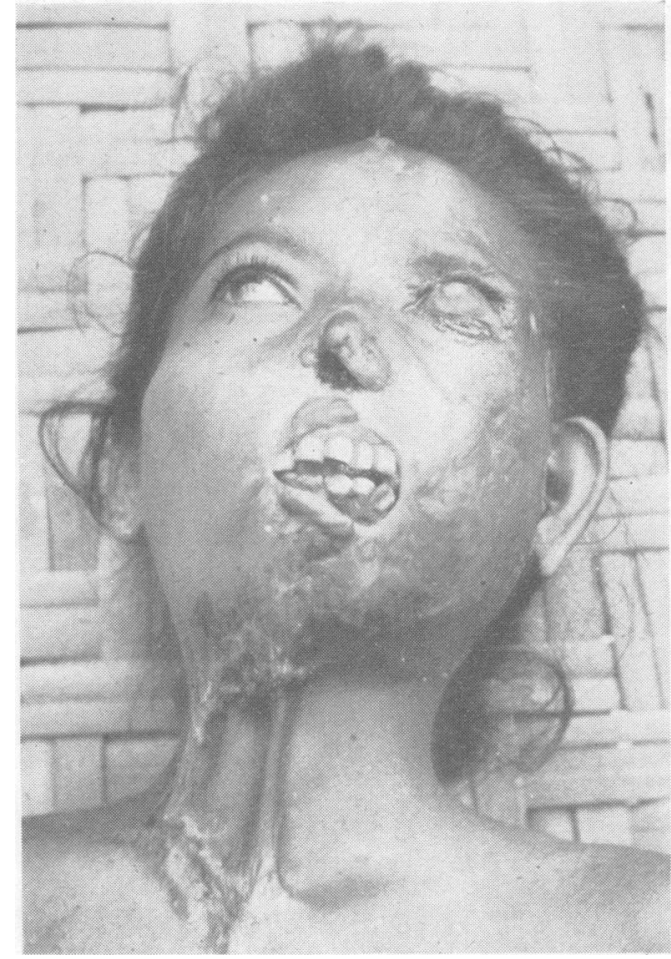

Fig. 14.-Rhinopharyngitis mutilans ("gangosa") of the late ulcerative stage of yaws.

\section{Schöbl stated:}

"Initial or metastatic yaws on mucous membranes are not known to exist; but direct spreading of primary or metastatic yaws from the skin across the direct muco-cutaneous border on to the mucous membranes is by no means an uncommon occurrence. This speaks in favour of my contention that the starting point of gangosa is the skin yaw or late ulcerating yaws lesion, extending directly on to the mucous membrane, ... that the histo-pathological process affects a very extensive area of the mucous membrane, far greater than the area which actually undergoes the real ulceration in any stage of the development of the condition,.... . by direct extension of skin lesions, either early (by super-infection) or late, on to the mucous membranes of the nose shows that this mode of pathology is the commonest if not the only one."
Since then no further experimental work has been conducted to increase our knowledge of ulcerations caused by framboesia alone, including those giving rise to the bone lesions which are not frequently seen in the field but are yet so important (Hackett, 1946). Experimentally, we have never seen bone lesions develop except from pre-existing skin lesions, which are encountered during the stage of metastatic generalization as well as during the later stages. Since treponemata have never been demonstrated in these bone lesions, or in the similar syphilitic lesions, it may be safe to assume that the bone changes are toxic, perhaps allergic. This view is supported by Chiari (1938), who described identical bone changes in children with long-standing pyodermia.

This "toxic" or "allergic" pathogenesis applies to the hyperkeratotic, often fissured, keratodermia of the soles of the feet and/or the palms of the hands in late yaws which is seen in over 50 per cent. of yaws patients in the field (Fig. 15). No treponemes are found in these lesions, which must be strictly differentiated from the common clavus thickening. They may be compared with the obviously "allergic" palmar and plantar hyperkeratoses occasionally observed in chronic gonorrhoea.

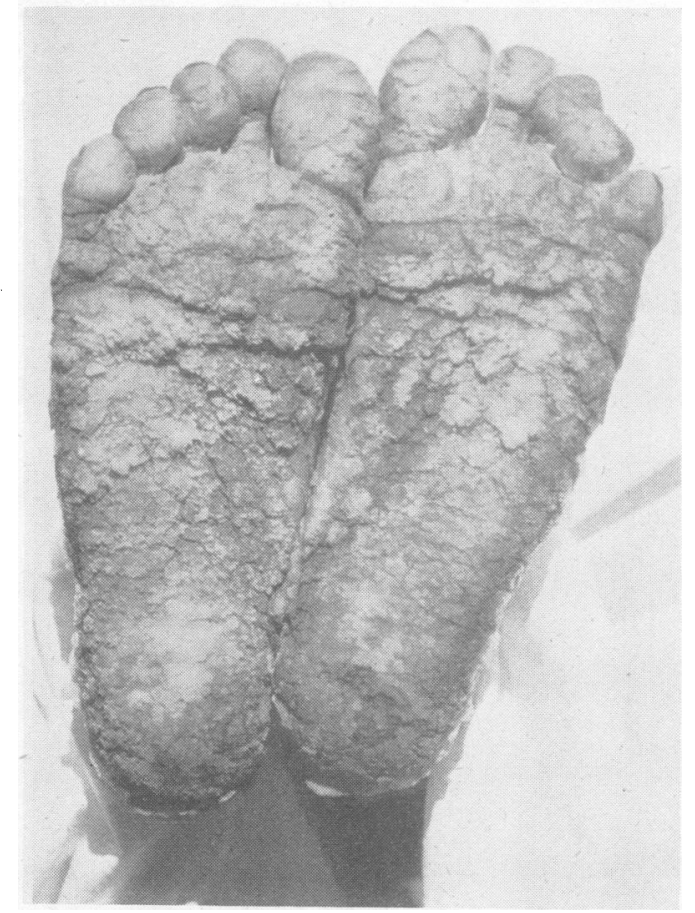

Fig. 15.-Keratodermia plantaris framboesica, with extensive hyperkeratosis and deep painful fissures. 
Histo-pathological changes in rhinopharyngitis mutilans (gangosa) occur, frequently because of the close ontogenic relationship of the mucous membranes of the facial orifices to the adjacent outer skin. In embryo-genesis, upgrowth of the mandibular arch and nasal and maxillary processes around the stomodeum forms the final facial contours. For a time, the ectodermal lining comes into direct contact with entoderm of the fore-gut, pressing aside the intervening mesoderm. Incidentally, gangosa pathology affects the mucosa only up to the area which coincides both with the pocket of Sessel and with the sensory border of the maxillary trigeminus ramus. As to intimal or medial pathology so typical of syphilitic ulcerations, Hallenberger (1916) stated that in gangosa, too, the blood vessels show no changes.

It is imperative to realize that the clinical morphology and the relevant histo-pathology of the initial "primary" yaws lesion are identical with those of the metastatic polypapillomata, whereas in syphilis both the morphology and the histopathological architecture are quite distinct in the primary syphilitic chancre and the various secondary syphilides.

\section{Pinta}

In this disease, the histo-pathological architecture is similar to that found in yaws, except that allergic exacerbation of existing skin pintides with ulcer formation never occurs. Hence, clinically, pinta efflorescences are not known to break down. In comparison with the histo-pathological changes in yaws, the inflammatory changes in pinta are of only very moderate degree. Acanthosis is only slight, and leucocytic diapedesis is minimal without the formation of intra-epidermal abscesses.

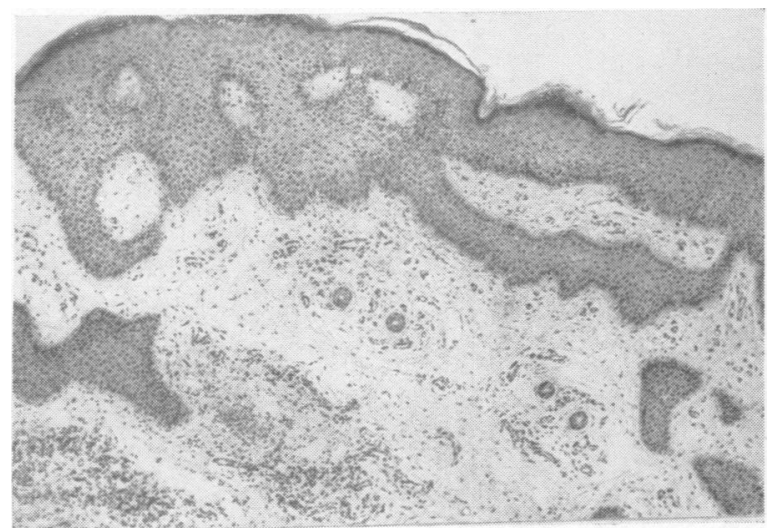

Fig. 16.-Pintide. Sl:ght parakeratosis, acanthosis, and spongiosis; moderate perivascular infiltrates in cutis. No pathology of intima or media.

(I am indebted for this biopsy material to Dr. Francisco I. Marquez, Mexico, D.F.).
In pinta, there is the same singular freedom from vascular pathology at all stages of the disease, and in all clinical manifestations. At most, in pintides of long standing, a slight swelling of some endothelial nuclei of the intima may be noticeable (Figs 16, 17, 18).

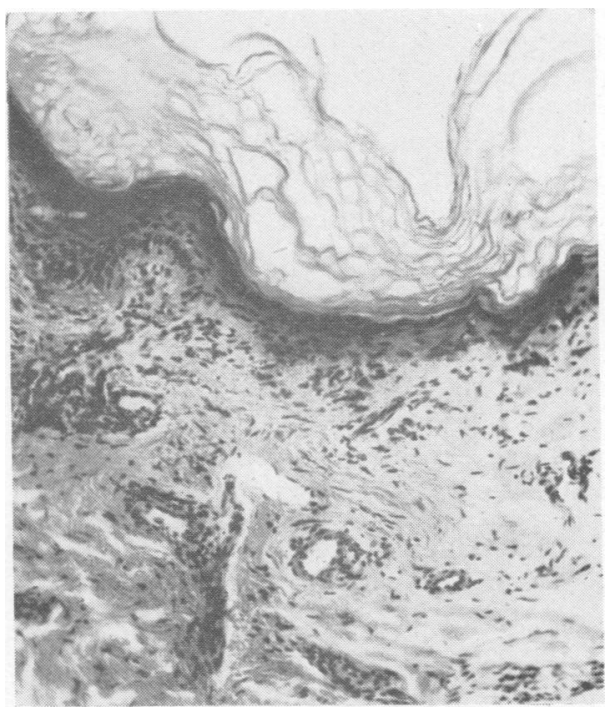

Fig. 17.-Scaly circinate pintide. Marked hyperkeratosis, atrophy of rete malpighi from pressure underneath of oedematous corium, with pathological perivascular infiltrate. Intact intima and media.

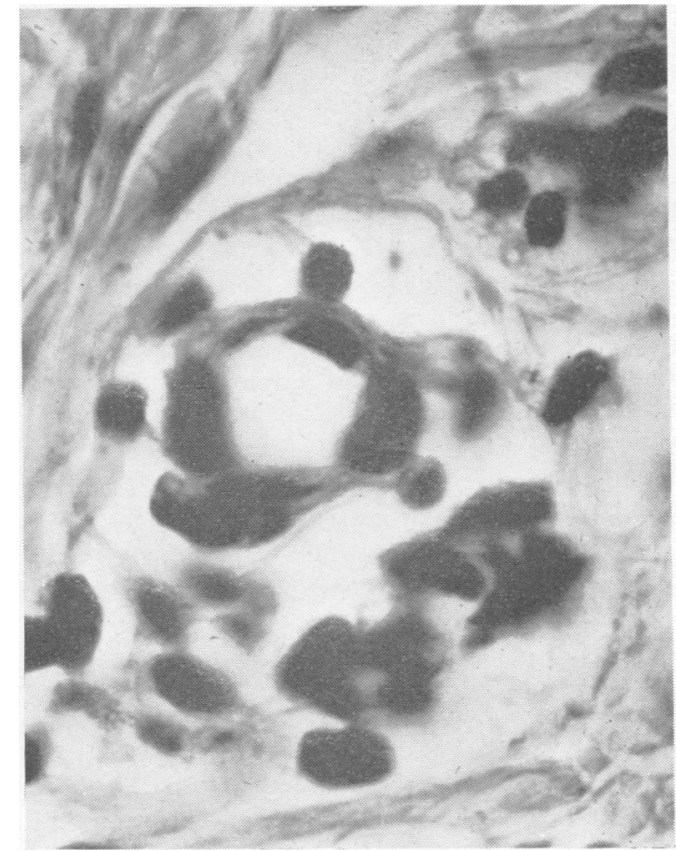

Fig. 18.-Pintide, high magnification showing only slight swelling of endothelial nuclei, without proliferation of intima, and no narrowing of capillary vessels. 


\section{Conclusion}

During both the early stages of yaws with the initial papular, or papillomatous lesion, and the so-called "secondary" framboesial period with metastatic polypapillomata, the characteristic tendency of $T$. pertenue to affect the upper layers of the skin only, without affecting the cardiovascular and central nervous systems, may be described as the "epidermiotropism" of yaws treponemes.

In this connexion it may not be amiss to point to the painstaking research of Williams (1935) and of Cole, Harkin, Kraus, and Moritz (1955) who found numerous examples of bone lesions on the American continents dating from pre-Columbian times, but none whatsoever in Europe, Africa, or Asia before 1494. As mentioned above, experimental evidence established that it is only in the later periods of the framboesial disease, when a definite allergic stage already exists, that any sudden increase in antigen production may result in an exacerbation of yaws lesions with subsequent ulcerations and the typical allergic histo-pathology.

In contrast, the well-known syphilitic pathology affecting both intima and media with proliferation of the intima, and with the narrowing and even obliteration of the vascular lumina (Heubner's endarteritis), is definite evidence of the preference of $T$. pallidum for the mesodermal layers, i.e. "mesodermotropism". Alternatively, inasmuch as T. pallidum invades and affects all layers, the term "panblastotropism" might be used for the treponema of syphilis.

Finally, since Weichbrodt and Jahnel (1919) pointed out that the treponemes are "frigitropic" and "thermophobic" (Bessemans, 1937), it has been established that:

experimental results suggest that the optimum temperature for the in vivo multiplication of treponemes is in the neighbourhood of $35^{\circ}$ to $37^{\circ} \mathrm{C}$. and that the development of treponemal lesions in a given tissue is related, in part at least, to the approximation of the local temperature to this level (Hollander and Turner, 1955).

Hence, apart from the distinct preference for the various skin layers of the treponemes causing syphilis, yaws, and pinta, the existing local temperature appears to condition, at least to some extent, both the appearance and clinical morphology of treponemal pathology.

In spite of the decline in the incidence of syphilis apparently due to the spectacular and still unfailing therapeutic efficacy of the penicillins, and to better social and follow-up conditions, many practical as well as scientific problems still remain regarding syphilis, as long as $T$. pallidum survives, and foci of infection persist. Not the least will be the increase of syphilis among people in the tropics where yaws, which had guaranteed a relative cross-immunity against naturally-acquired syphilis, has now been so much reduced as a result of successful mass treatment campaigns (Manson-Bahr, 1954).

\section{Summary}

(1) The comparative histo-pathology of syphilis, yaws, and pinta is discussed.

(2) The distinct tendency of both the $T$. pertenue of yaws, and of the $T$. pinta (carate) of pinta to affect the upper layers of the skin only, without affecting the cardiovascular and central nervous systems, and the non-existence of congenital yaws or pinta may be described as "epidermiotropism". By contrast, the well-known syphilitic changes of the inner organs and the cardiovascular and central nervous systems are indicative of what may be termed the "mesodermotropism" or "panblastotropism" of $T$. pallidum.

(3) This different biological behaviour in the tissues of the host largely explains the differences in the clinical and pathological symptoms and lesions in these diseases.

\section{REFERENCES}

Bessemans, J. F. A. (1930). Urol. cutan. Rev., 34, 71.

Chiari, O. von (1938). Arch. Kinderheilk., 115, 149

Cole, H. N., Harkin, J. C., Kraus, B. S., and Moritz, A. R. (1955). A.M.A.Arch. Derm. 71, 231.

Goodpasture, E. W. (1923). Philipp. J. Sci., 22, 263.

Hackett, C. J. (1946). Trans. roy. Soc. trop. Med. Hyg., 40, 206.

Hallenberger, - (1916). Arch. Schiffs-u. Tropenhyg., 20, Beiheft 3.

Hasselmann, C. M. (1930). Zbl. Haut-u. GeschlKr., 33, 273.

(1931). China med. J., 45, 1131 .

(1931). China med. J., 45,

(1933). China med. J., 47, 584

(1938a). Ber. phys. med. Ges. Wurzburg (1937), n.s. 61, 51.

(1938b). Arch. Derm. Syph. (Chicago), 38, 837.

(1949). Arch. Derm. Syph. (Berl.), 189, 340.

(1951). O.S.R. News (Indonesia), 3, 156

(1952). A.M.A. Arch. Derm. Syph., 66, 107.

(1953a). J. sci. Res. (Djakarta), 2, 39.

(1953a). J. sci. Res. (Djakarta), 2,

(1953b). Amer. J. Syph., 37, 151.

(1953c). Z. Haut-u. GeschlKr., 15, 347.

Hollander, D. H., and Turner, T. B. (1954.). Amer. J. Syph., 38, 489.

McLeod, C., and Turner, T. B. (1946). Ibid., 30, 442.

Manson-Bahr, P. E. C. (1954). Trans. roy. Soc. trop. Med. Hyg., 48, 242.

Schöbl, O. (1928). Philipp. J. Sci., 35, 209.

and Hasselmann, C. M. (1932). Arch. Schiffs-u. Tropenhyg., 36, Beiheft 2.

and Sellards, A. W. (1926). Philipp. J. Sci., 31, 1.

,-- , and Lacy, G. R. (1926). Ibid., 30, 475 .

Sellards, A. W., Lacy, G. R., and Schöbl, O. (1926). Ibid., 30, 463. Turner, T. B. (1937). Amer. J. Hyg., 25, 477.

, Hollander, D. H., and Schaeffer, K. (1953). Bull. Wld Hlth Org., $8,7$.

Weichbrodt, R., and Jahnel, F. (1919). Dtsch. med. Wschr., 45, 483. Williams, H. U. (1935). Arch. Path. (Chicago), 20 :596. 